\title{
Contrast Transfer and Noise Minimization in Electron Ptychography
}

Colum M. O’Leary ${ }^{1}$, Gerardo T. Martinez ${ }^{1}$, Emanuela Liberti ${ }^{1,2}$, Martin J. Humphry ${ }^{3}$, Angus I. Kirkland $^{1,2}$ and Peter D. Nellist ${ }^{1}$

1. University of Oxford, Department of Materials, Oxford, UK.

2. electron Physical Sciences Imaging Centre (ePSIC), Diamond Light Source, UK.

3. Phasefocus Ltd, Sheffield, UK.

The development of electron ptychography using fast pixelated detectors (FPDs) has enabled localized high contrast imaging of light-element crystalline and quasi-crystalline structures at atomic resolution. As a result, simultaneous images of light- and heavy-elements can be routinely obtained from the same experiment [1]. However, the application of such methods to more complex materials with longer-range ordering, many of which are sensitive to the electron beam, has been less frequently reported. This area of research is ever more pertinent since the success of cryo-electron microscopy for biological applications.

When performing phase-imaging of beam-sensitive materials, reducing the electron dose (electrons per unit area) or dose rate (dose per unit time) incident upon the sample is essential, but it is also crucial to optimize information transfer for all spatial frequencies. The conventional measure for this is the phase contrast transfer function (PCTF), which has been well-developed for non-iterative electron ptychography methods [2]. However, unlike conventional TEM or bright-field STEM imaging methods, the information transfer of 4D STEM data is only noise-limited, and the intrinsic contrast transfer of 4D STEM imaging methods is purely dependent upon the applied post-processing phase- (or field-) retrieval algorithm.

In this contribution, we explore how one can optimize information transfer while minimizing noise effects in STEM ptychography phase reconstructions. Firstly, we analyze the intrinsic PCTF for noniterative electron ptychography and its effect on phase reconstructions for (a) single atom simulations, (b) amorphous carbon (experiment), (c) the edge of a graphene monolayer and (d) a functionalized carbon nanotube (experiment) [1]. Secondly, we demonstrate the effects of normalizing the PCTF with respect to the noise and derive a Fourier signal-to-noise ratio (FSNR) as a function of spatial frequency.

Figure 1 shows the ptychographic reconstructions with (a) a conventional PCTF, (b) a flat PCTF and (c) a noise-normalized PCTF for a graphene monolayer edge. The 4D STEM data was acquired with the JEOL 4DCanvas installed on the JEOL ARM200F at University of Oxford (HT $=80 \mathrm{kV}, \alpha=22.48$ $\mathrm{mrad}$ ). The corresponding PCTFs (inset) represent the relative strengths of information transfer for each spatial frequency allowed by the reconstruction.

With reference to a recent study [3], we compare intrinsic and noise-normalized PCTFs of several phase- and field-contrast imaging methods (integrated differential phase-contrast (iDPC), non-/iterative ptychography, etc.) for an array of experimental examples. These results will be discussed in an attempt to quantify a 'noise threshold' for electron ptychography methods. 


\section{References:}

[1] H Yang et al, Nature Communications 7 (2016), p. 12532.

[2] H Yang, TJ Pennycook and PD Nellist, Ultramicroscopy 151 (2015), p. 232-239.

[3] T Seki, Y Ikuhara and N Shibata, Ultramicroscopy 193 (2018), p. 118-125.

[4] The financial support of JEOL (UK) Ltd and the EPSRC is gratefully acknowledged.

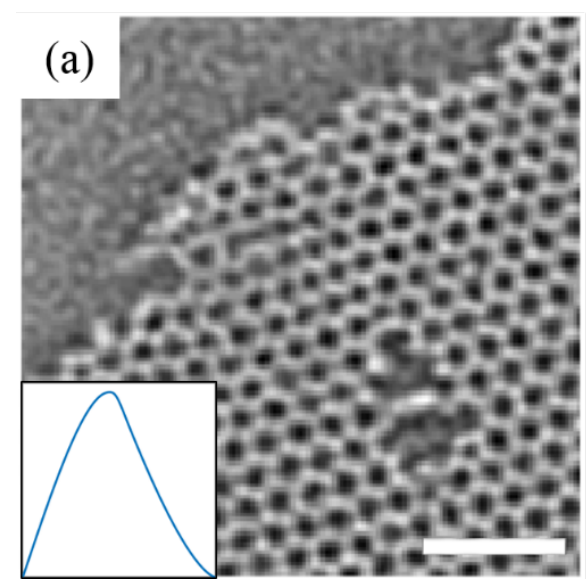

(b)

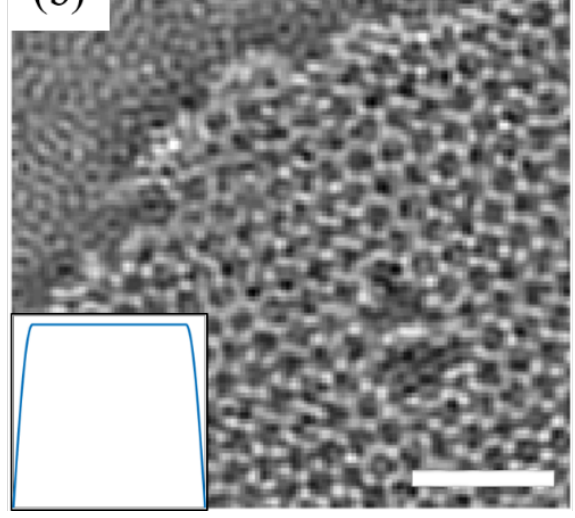

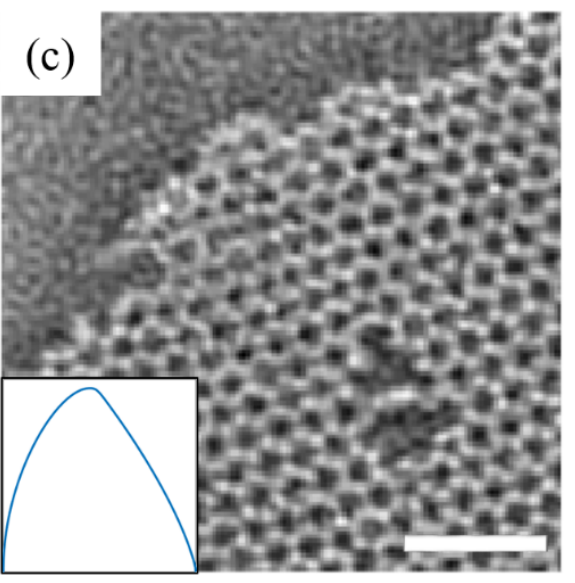

Figure 1. Phase reconstructions of a graphene monolayer edge using electron ptychography for (a) intrinsic contrast transfer, (b) flat contrast transfer and (c) noise-normalized contrast transfer. Insets correspond to respective phase contrast transfer functions (horizontal axis: $0-2 \alpha$, vertical axis: 0 - 1.05). Scale bar: $1 \mathrm{~nm}$. 